\title{
Simulation of Cerebral Aneurysm Growth and Prediction of Evolving Rupture Risk
}

\begin{abstract}
Martin Kroon
Department of Solid Mechanics, Royal Institute of Technology, Osquars Backe 1, 10044 Stockholm, Sweden

Correspondence should be addressed to Martin Kroon, martin@hallf.kth.se

Received 2 March 2011; Accepted 2 May 2011

Academic Editor: Xiaosheng Gao

Copyright () 2011 Martin Kroon. This is an open access article distributed under the Creative Commons Attribution License, which permits unrestricted use, distribution, and reproduction in any medium, provided the original work is properly cited.

Cerebral aneurysms are local expansions of blood vessel walls in the brain blood system. The rupture of an aneurysm is a very severe event associated with a high rate of mortality. When cerebral aneurysms are detected, clinicians need to decide if operation is required. The risk of aneurysm rupture is then compared to the risks associated with the medical intervention. In the present paper, a probabilistic framework for a mechanically based rupture risk assessment of cerebral aneurysms is proposed. The method is based on the assumption that the strength of aneurysmal tissues can be described by a statistical distribution. A structural analysis of the aneurysm in question is performed, and the maximum stress experienced by the aneurysm is compared to the strength distribution. The proposed model was compared with clinical results for ruptured aneurysms in terms of rupture density and accumulated rupture risk as a function of aneurysm size. The model was able to reproduce the clinical results well. The proposed framework may potentially be used under in vivo conditions to predict the risk of rupture for diagnosed aneurysms.
\end{abstract}

\section{Introduction}

Cerebral (intracranial) aneurysms are local expansions on arteries in the cerebral blood vessel system, and these baloon-like structures may reach a size of over $30 \mathrm{~mm}$ in diameter [1]. Aneurysms are a fairly common pathology in humans [2], and intracranial aneurysms are more prevalent in females than in males [3]. Most bifurcations of the cerebral vasculature are structurally stable, but a small number develop a weakness that causes the wall to expand outwardly in the region near the flow divider of the branching artery [4-6]. Not much is known about the etiology of aneurysms. Once detected, the changes of the aneurysmal wall are, in general, already advanced, and the wall may also be affected by atherosclerosis [7]. Genetic factors are believed to play a role in the pathogenesis of intracranial aneurysms [8], but other factors, such as hemodynamic stress at arterial bifurcations, congenital defects, degenerative arterial wall changes, smooth muscle cell apoptosis, smoking, and excessive alcohol consumption, are also believed to contribute to aneurysmal development [9-13].

Unruptured cerebral aneurysms may give different kinds of symptoms, including headache, orbital pain, and loss of vision [14], but in most cases they remain asymptomatic. The mortality and morbidity rates associated with the rupture of intracranial aneurysms and the resulting subarachnoid hemorrhage are very high. About $50 \%$ of patients with ruptured intracranial aneurysms die within one month after the event. Of those who survive, more than one third have major neurologic deficits $[8,15]$. When rupture occurs, the point of rupture is generally in the dome of the aneurysm $[4,8]$. Intracranial aneurysms do not normally rupture if smaller than $10 \mathrm{~mm}$ in diameter $[4,16,17]$. The risk of rupture for diagnosed aneurysms is mainly based on the size, growth rate, morphology, and location [14], and cerebral aneurysms are usually not treated if smaller than $5 \mathrm{~mm}$ in size.

When cerebral aneurysms are detected, clinicians need to assess the risk of rupture and if operation is needed. The risk of rupture is then weighed against the risks inherent in the operation methods. Cerebral aneurysms are treated either by surgical clipping or by endovascular treatment $[18,19]$. Surgical clipping means that the skull of the patient is opened, the aneurysm is exposed, and a small metallic clip is inserted to shield off the aneurysm sac from the pulsating blood flow. Endovascular treatment is a less severe intervention. 
A thin coil is then inserted through the blood system into the aneurysm sac where it curls and stimulates the formation of a thrombus in the sac. The effect of this thrombus is also to shield off the aneurysm sac from the blood flow.

When assessing the risk of aneurysm rupture, some kind of probabilistic framework should be aimed for. Clinical studies have been performed to clarify how the risk of rupture correlates with, for example, aneurysm size, aneurysm location, and also the age, gender, and ethnicity of patients $[1,14,20,21]$. However, since rupture of an aneurysm is a mechanical phenomenon, the assessment of the rupture risk should be performed on the basis of the mechanical fields (stress, strain) in the aneurysm. From a mechanical point of view, rupture implies that the stress imposed on the aneurysm wall exceeds its mechanical strength. The details of the failure mechanisms of intracranial aneurysmal tissue are, however, not well understood. Some studies indicate that aneurysm rupture is the result of a quite complex interaction between mechanical and biochemical processes [22]. For example, ruptured aneurysms have been observed to exhibit more significant endothelial damage, stronger inflammatory cell invasion, more structural changes in the vessel wall, and a higher activity of elastase and collagenase (fibre-degrading proteins) compared with unruptured aneurysm tissue [14].

Some measurements of the macroscopic mechanical properties of cerebral arteries and aneurysms exist [23-29], and the structural organisation of these tissues is fairly well documented [5, 6, 30-40]. However, in contrast to many engineering materials, the wall of an aneurysm is a living and metabolising structure, able to add to and reinforce itself. As a result, aneurysms may enlarge, but this does not necessarily imply thinning, because new material is continuously added as the aneurysm expands.

For metallic materials, several schemes and models are available for probabilistic assessments of the failure risk, for example, [41-46]. In the present paper, a tentative probabilistic framework is proposed in which the rupture risk for cerebral aneurysms is assessed based on the mechanical fields in the aneurysm wall. To the author's knowledge, this is the first attempt to use the mechanical fields of cerebral aneurysms to assess the risk of rupture probabilistically. The methodology includes a statistical distribution of the mechanical strength of the aneurysmal tissue and a structural analysis of the aneurysm. The maximum principal stress in the aneurysm wall is compared to the strength distribution, and based on this, a risk of rupture is obtained. For the constitutive behaviour of the aneurysmal tissue the model proposed by [47] is adopted. In Section 2, the proposed probabilistic framework is outlined. A theoretical model of an idealised aneurysm is presented in Section 3. The probabilistic framework is then assessed in Section 4, where the predicted rupture risk is compared to clinical observations. The findings are discussed in Section 5.

\section{Probabilistic Framework}

Consider the saccular cerebral aneurysm in Figure 1. Blood is continuously pumped through the parent artery, and during the cardiac cycle, the static pressure in the aneurysm

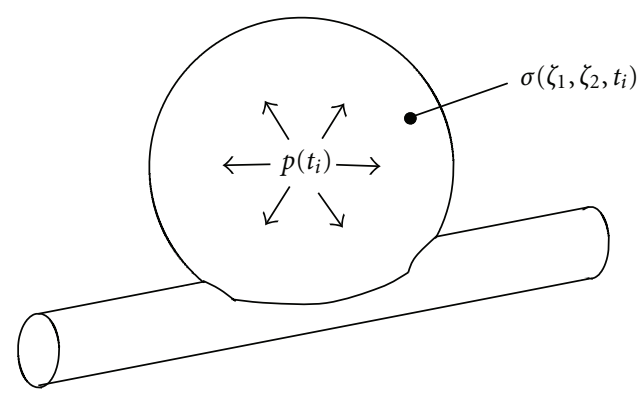

FIGURE 1: A saccular cerebral aneurysm exposed to an internal pressure $p\left(t_{i}\right)$ caused by the pulsating blood flow in the parent artery. The internal pressure $p\left(t_{i}\right)$ causes a stress state in the aneurysm wall, characterised by the Cauchy stress tensor $\sigma\left(\zeta_{1}, \zeta_{2}, t_{i}\right)$. The surface geometry of the aneurysm is parameterised by the two convected coordinates $\zeta_{1}$ and $\zeta_{2}$.

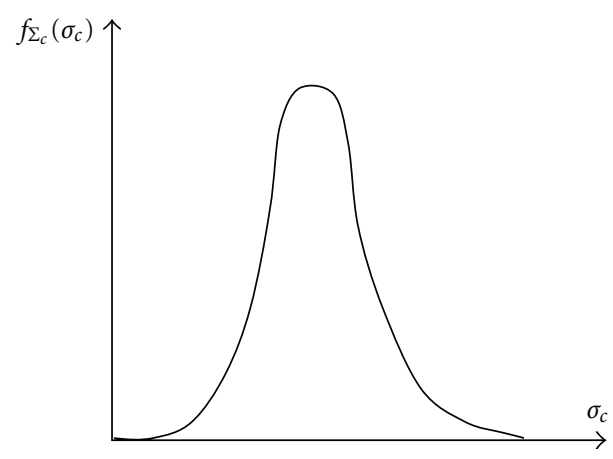

FIGURE 2: Density function $f_{\Sigma_{c}}\left(\sigma_{c}\right)$ for stochastic variable $\Sigma_{c}$ characterising the statistical scatter in strength of the aneurysm tissue.

varies according to $p\left(t_{i}\right)$. This varying pressure gives rise to a varying stress state in the aneurysm wall, characterised by the Cauchy stress $\boldsymbol{\sigma}\left(\zeta_{1}, \zeta_{2}, t_{i}\right)$. The time variable $t_{i}$ is associated with processes that take place during a cardiac cycle. Cerebral aneurysms are usually relatively thin, and the geometry of the aneurysm sac is therefore described as a membrane with two convected surface coordinates $\zeta_{1}$ and $\zeta_{2}$. The maximum principal stress is denoted $\sigma_{1}=$ $\sigma_{1}\left(\zeta_{1}, \zeta_{2}, t_{i}\right)$. During the cardiac cycle, the internal pressure $p$ reaches its peak at systole. This causes an associated peak in maximum principal stress in the aneurysm wall denoted $\sigma_{\max }=\max \left(\sigma_{1}\left(\zeta_{1}, \zeta_{2}, t_{\mathrm{i}}\right)\right)$.

Tensile tests performed on cerebral aneurysm tissue indicate that the strength of the tissue shows a considerable statistical scatter $[5,28,48,49]$. The strength of the tissue, $\sigma_{c}$, may therefore be characterised by a stochastic variable $\Sigma_{\mathrm{c}}$ with a density function $f_{\Sigma_{c}}\left(\sigma_{c}\right)$; see Figure 2 . Several options for $f_{\Sigma_{c}}\left(\sigma_{c}\right)$ exist, for example, the normal distribution and the Weibull distribution.

We assume that, for a specific aneurysm sac, the same strength distribution may be applied for the whole sac. In general, the material strength will vary over the aneurysm sac, but, by applying a strength distribution that is valid for the weakest parts of the sac, a conservative rupture risk 
assessment is attained. The probability of aneurysm rupture, $P_{\text {rup }}$, may then be expressed as

$$
P_{\text {rup }}=F_{\Sigma_{c}}\left(\sigma_{\max }\right)=\int_{0}^{\sigma_{\max }} f_{\Sigma_{c}}\left(\sigma_{c}\right) d \sigma_{c},
$$

where $F_{\Sigma_{c}}\left(\sigma_{c}\right)$ is the distribution function of the stochastic variable $\Sigma_{c}$.

Thus, if the statistical distribution of the strength of aneurysmal tissue $f_{\Sigma_{c}}\left(\sigma_{c}\right)$ is known, and if the geometry, boundary conditions, and constitutive behaviour of a specific aneurysm are known, the risk of rupture and subarachnoid hemorrhage $P_{\text {rup }}$ can be estimated using the scheme above.

\section{Aneurysm Model}

3.1. Continuum Mechanics Framework. The continuum mechanics framework now introduced serves as a basis for the constitutive model to be presented in Section 3.2. A more rigorous description of this framework is provided in [47].

Due to the relative thinness that aneurysmal tissues normally display, the aneurysmal tissue is here characterised as a membrane. A reference frame of right-handed, rectangular coordinate axes at a fixed origin with orthonormal basis vectors $\mathbf{e}_{i}, i=1,2,3$ is defined. The position vector $\mathbf{X}$ in the reference configuration $\Omega_{0}$ is given as $\mathbf{X}=X_{i} \mathbf{e}_{i}$, where $X_{i}$ are the referential coordinates. The position vector $\mathrm{x}$ in the current configuration $\Omega$ is $\mathbf{x}=x_{i} \mathbf{e}_{i}$, where $x_{i}$ denote the related spatial coordinates. The same reference frame is used for the reference and current configurations. The displacement vector $\mathbf{u}$ is then defined as $\mathbf{u}=\mathbf{x}-\mathbf{X}=$ $u_{i} \mathbf{e}_{i}$. Material points on the membrane (aneurysm surface) are labeled by the surface convected coordinates $\zeta_{1}$ and $\zeta_{2}$, together with $\zeta_{3}$ oriented normal to the membrane surface. Greek indices are used to denote the quantities measured using the membrane intrinsic metric. The associated basis vectors $\mathbf{a}_{1}, \mathbf{a}_{2}$, and $\mathbf{a}_{3}$ define a local Euclidean frame on the membrane. The deformation gradient $\mathbf{F}$ is defined according to

$$
\mathbf{F}=\frac{\partial x_{i}}{\partial \zeta_{\alpha}} \mathbf{e}_{i} \otimes \mathbf{a}_{\alpha}
$$

and the right Cauchy-Green tensor is $\mathbf{C}=\mathbf{F}^{\mathrm{T}} \mathbf{F}$.

The loading imposed on the aneurysm is caused by the blood pressure $p$ in the parent artery. The pressure in a blood vessel varies in a pulsating manner, where the lowest pressure (at diastole) is denoted $p_{\text {dia }}$ and the highest pressure (at systole) is denoted $p_{\text {sys. }}$. We consider four different deformed configurations. Three of these configurations, $\Omega_{\mathrm{lf}}, \Omega_{\mathrm{dia}}$, and $\Omega_{\text {sys }}$, are associated with the applied pressure loads $p=0^{+}$, $p=p_{\text {dia }}$, and $p=p_{\text {sys }}$, respectively. The configuration $\Omega_{\text {lf }}$ defines the load-free state of the aneurysm when an infinitesimally small pressure $p=0^{+}$is applied. Collagen is the dominant structural component in aneurysm walls, and since collagen fibres are continuously deposited as the aneurysm evolves and grows, fibres, deposited at different times, will have different natural configurations. In order to account for this, we also introduce a fourth deformed configuration, $\Omega_{\mathrm{dep}}$, at which a specific fibre is deposited.
The deformation gradient $\mathbf{F}$ is evaluated in the four deformed configurations $\Omega_{\mathrm{lf}}, \Omega_{\mathrm{dia}}, \Omega_{\mathrm{sys}}$, and $\Omega_{\mathrm{dep}}$, giving rise to the entities $\mathbf{F}_{\mathrm{lf}}, \mathbf{F}_{\text {dia }}, \mathbf{F}_{\text {sys }}$, and $\mathbf{F}_{\text {dep }}$, respectively. The deformation gradients $\mathbf{F}_{\mathrm{dia}}^{\star}=\mathbf{F}_{\mathrm{dia}} \mathbf{F}_{\mathrm{lf}}^{-1}$ and $\mathbf{F}_{\mathrm{sys}}^{\star}=\mathbf{F}_{\mathrm{sys}} \mathbf{F}_{\mathrm{lf}}^{-1}$ denote the deformations experienced by the aneurysmal wall from the load-free configuration $\Omega_{\text {If }}$ to the diastolic $\Omega_{\text {dia }}$ and systolic $\Omega_{\text {sys }}$ states, respectively. The entity $\mathbf{F}_{\mathrm{cc}}=\mathbf{F}_{\mathrm{sys}} \mathbf{F}_{\mathrm{dia}}^{-1}$ is the deformation from the diastolic state $\Omega_{\text {dia }}$ to the systolic state $\Omega_{\mathrm{sys}}$, quantifying the cyclic deformation of the tissue during the cardiac cycle (the index "cc" "stands for "cardiac cycle"). For all of the deformation gradients defined above, dependence on time and position is understood.

The deformation gradient $\mathbf{F}_{\text {dep }}=\mathbf{F}\left(t_{\text {dep }}\right)$ is the deformation at time $t_{\text {dep }}$ at which a specific fibre is deposited, and the deformation $\mathbf{F}_{\text {sys }}^{\prime}=\mathbf{F}_{\text {sys }} \mathbf{F}_{\text {dep }}^{-1}$ quantifies the subsequent deformation experienced by this particular fibre in the systolic state.

3.2. Constitutive Model. The constitutive model for aneurysmal tissue has been presented elsewhere [47], and only a brief outline is given here. The wall of the saccular cerebral aneurysm is modelled as a hyperelastic membrane, whose constitutive behaviour is governed by a $3 \mathrm{D}$ strain-energy function $\Psi$. We assume that collagen is the only load-bearing constituent in the aneurysmal wall, which is taken to be a development of the adventitia of the original healthy arterial wall. The continuous turnover of collagen is the driving mechanism for aneurysmal remodelling and growth. This remodelling and turnover of collagen is assumed to be accomplished by fibroblasts (fibre-producing cells), which are spread throughout the collagen network.

The structure of the aneurysm wall is taken to be made up of $n$ discrete and distinct layers of collagen fibres (plies that form a laminate). Within a layer (ply) with index $i$, the collagen fibres and the embedded fibroblasts are perfectly aligned in a direction $\phi_{i}=\pi(i-1) / n$, defined with respect to the local reference coordinate system $\zeta_{1}-\zeta_{2}$, where $\phi_{1}$ is directed in the $\zeta_{1}$-direction. The aneurysmal wall is assumed to have a constant total initial thickness $H_{0}$ in $\Omega_{0}$ (corresponding to the thickness of the adventitia of the parent artery), and each collagen layer is assigned an initial thickness of $H_{0} / n$.

The total strain energy $\Psi(t)$ per unit reference volume is computed by integration according to

$$
\begin{aligned}
\Psi(t) & =\frac{1}{n} \sum_{i=1}^{n} \Psi_{i}(t) \\
& =\frac{1}{n} \sum_{i=1}^{n} \int_{-\infty}^{t} g\left(t, t_{\mathrm{dep}}\right) \dot{m}_{i}\left(t, t_{\mathrm{dep}}\right) \psi_{\mathrm{fib}}\left(t, t_{\mathrm{dep}}\right) \mathrm{d} t_{\mathrm{dep}},
\end{aligned}
$$

where $\Psi_{i}(t)$ is the strain energy of layer $i$. The life cycle function $g$ (cf. [56]) accounts for the turnover of the collagen fibres, and a simple pulse function $g\left(t, t_{\text {dep }}\right)=\Theta\left(t-t_{\text {dep }}\right)-$ $\Theta\left(t-t_{\mathrm{dep}}-t_{\mathrm{cl}}\right)$ is used, where $\Theta(t)$ is the Heaviside step function and $t_{\mathrm{cl}}$ is the life-time of the collagen fibres. The time variable $t$ is associated with processes that take place over multiple cardiac cycles, such as tissue remodelling. 
The mass production rate of collagen per unit reference volume in layer $i$, denoted $\dot{m}_{i}(t)$, is expressed as

$$
\dot{m}_{i}(t)=n_{\mathrm{fb}}(t) \beta_{0}\left(C_{\mathrm{cci}}\right)^{\alpha} .
$$

The collagen production rate $\dot{m}_{i}(t)$ depends on the current concentration of fibroblasts $n_{\mathrm{fb}}(t)$ and the cyclic deformation of these cells $C_{\mathrm{cci}}$. The cyclic deformation of fibroblasts is quantified by $\mathbf{C}_{\mathrm{cc}}=\mathbf{F}_{\mathrm{cc}}^{T} \mathbf{F}_{\mathrm{cc}}$. The scalar $C_{\mathrm{cc} i}$ is then defined as $C_{\mathrm{cci}}=\mathbf{C}_{\mathrm{cc}}: \mathbf{A}\left(\phi_{i}\right)$, where $\mathbf{A}\left(\phi_{i}\right)=\mathbf{M} \otimes \mathbf{M}$ is a structure tensor and $\mathbf{M}$ is a vector with components $M_{1}=\cos \phi_{i}, M_{2}=\sin \phi_{i}, M_{3}=0$ defining the direction of the fibres in layer $i$ in the reference configuration. The influence of the scalar $C_{\mathrm{cc} i}$ on the collagen production rate is modulated by the exponent $\alpha$. The parameter $\beta_{0}$ may roughly be interpreted as the mean collagen production rate per fibroblast of a healthy adventitia.

The current number of fibroblasts per unit reference volume is taken to be constant through the tissue thickness and is expressed as $n_{\mathrm{fb}}(t)=n_{\mathrm{fb} 0} J_{\mathrm{lf}}(t)$, where $n_{\mathrm{fb} 0}=$ $n_{\mathrm{fb}}(t=0)$ is the concentration of fibroblasts in a healthy (nonaneurysmal) adventitia and $J_{\text {lf }}=\operatorname{det} \mathbf{F}_{\text {lf }}$. It is assumed that collagen fibres, produced at time $t_{\mathrm{dep}}$, are inserted in the configuration $\Omega_{\text {sys }}$, implying that $\mathbf{F}_{\text {dep }}=\mathbf{F}_{\text {sys }}\left(t_{\text {dep }}\right)$. Collagen fibres, deposited at time $t_{\mathrm{dep}}$, are deposited in the configuration $\Omega_{\text {sys }}$ with a constant prestretch $\lambda_{\text {pre }}$. The total deformation $C_{\mathrm{fib}}$ of the fibres in the layer $i$, deposited at time $t_{\mathrm{dep}}$, can then be expressed as $C_{\mathrm{fib}}=\lambda_{\text {pre }}^{2} \mathbf{C}^{\prime}: \mathbf{A}\left(\phi_{i}\right)$, where $\mathbf{C}^{\prime}=\mathbf{F}_{\text {sys }}^{\prime} T \mathbf{F}_{\text {sys }}^{\prime}$. The strain energy $\psi_{\text {fib }}$ per unit mass stored in the fibres is expressed as $\psi_{\mathrm{fib}}=\mu\left(C_{\mathrm{fib}}-1\right)^{3}$, where $\mu>0$ is a positive material parameter, associated with the stiffness of collagen fibres. This expression is only valid when the fibres are in tension or are unloaded $\left(C_{\mathrm{fb}} \geq 1\right)$, whereas the fibres are assumed to have zero stiffness in compression $\left(C_{\mathrm{fib}}<1\right)$.

Membrane stresses are represented by a modified 2D second Piola-Kirchhoff stress tensor, with components defined as $S_{\alpha \beta}^{\star}=2 \partial \Psi / \partial C_{\alpha \beta}^{\star}$ with $\mathbf{C}^{\star}=\mathbf{F}^{\star T} \mathbf{F}^{\star}$. Since we consider a membrane, we only evaluate in-plane stresses, and $\alpha$ and $\beta$ therefore only take on the values 1 and 2. A modified Cauchy stress $\sigma_{\alpha \beta}^{\star}$ is also defined as

$$
\sigma_{\alpha \beta}^{\star}=\frac{H_{0}}{H_{\mathrm{lf}} J^{\star}} F_{\alpha \gamma}^{\star} S_{\gamma \delta}^{\star} F_{\delta \beta}^{\star},
$$

where $J^{\star}=\operatorname{det} \mathbf{F}^{\star}$ and $H_{\text {lf }}$ is the current tissue thickness in the load-free state $\Omega_{\mathrm{lf}}$. In the deformation from $\Omega_{\mathrm{lf}}$ to the diastolic and systolic states we assume incompressibility, which requires that $J^{\star} \equiv 1$. The tissue thickness will change as collagen is produced and degraded, and the current thickness in the load-free configuration, $H_{\mathrm{lf}}$, can be estimated as

$$
H_{\mathrm{lf}}=\frac{H_{0}}{n \lambda_{1} \lambda_{2}} \sum_{i=1}^{n} \frac{m_{i}}{m_{0}}
$$

where $m_{i}$ and $m_{0}$ denote the current and initial collagen mass content, respectively, and $\lambda_{1}$ and $\lambda_{2}$ are the in-plane principal stretches in the load-free state $\Omega_{\mathrm{lf}}$. The determinant $J_{\text {lf }}$ may then consequently be estimated as $J_{\text {lf }}=H_{\mathrm{lf}} \lambda_{1} \lambda_{2} / H_{0}$.

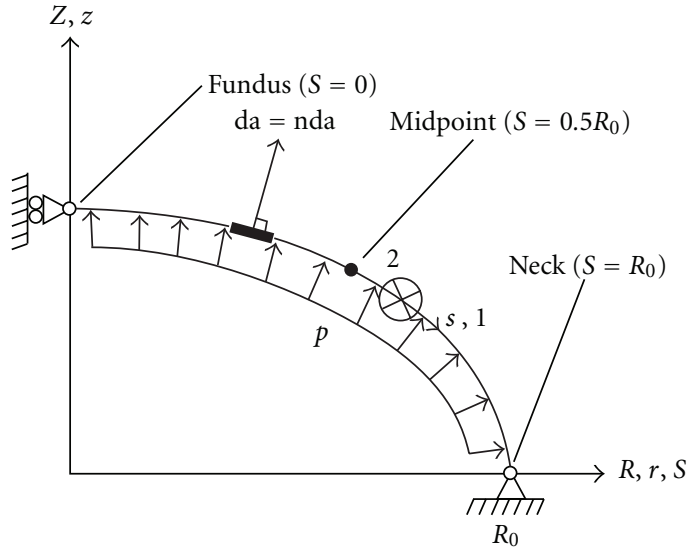

Figure 3: Profile of the membrane, definitions of coordinates and principal directions, and loading and boundary conditions.

3.3. Problem Formulation for Idealised Aneurysm. A saccular cerebral aneurysm is here modelled as an axisymmetric membrane, which is hinged along its periphery and exposed to a (blood) pressure $p$. The membrane formulation used here is based on a work by [50] (also utilised by [47, 51, $52]$ ), and a brief review of the formulation is provided below.

Consider the axisymmetric membrane, as illustrated in Figure 3. The surface profile can be parameterised using coordinates $S$ and $s$ in the reference and current configurations, respectively. Coordinates $R(S), Z(S), r(s)$, and $z(s)$ denote cylindrical coordinates in the reference and current configurations, respectively. The membrane is hinged at $R=$ $R_{0}$. Boundary conditions are thus imposed according to

$$
\begin{gathered}
R=0: r=0, \\
R=R_{0}: r=R_{0}, \quad z=0 ;
\end{gathered}
$$

see Figure 3.

Principal directions 1 and 2 coincide with the direction of $s$ and the circumferential direction, respectively, as indicated in Figure 3. The convected surface coordinates $\zeta_{1}$ and $\zeta_{2}$ are taken to coincide with the principal directions 1 and 2 in Figure 3, respectively. The principal stretches in the plane of the membrane can be expressed as

$$
\lambda_{1}=\frac{\mathrm{d} s}{\mathrm{~d} S}=\sqrt{\left(\frac{\mathrm{d} r}{\mathrm{~d} S}\right)^{2}+\left(\frac{\mathrm{d} z}{\mathrm{~d} S}\right)^{2}}, \quad \lambda_{2}=\frac{2 \pi r}{2 \pi R}=\frac{r}{R} .
$$

The potential energy $\Pi$ of the membrane, inflated by a given constant pressure $p$, is

$$
\begin{aligned}
\Pi & =\int_{\Omega_{0}} \Psi \mathrm{d} V-p \int_{\partial \Omega_{\sigma}} \mathbf{u} \cdot \mathrm{d} \mathbf{a} \\
& =\pi \int_{0}^{R_{0}}\left(2 R \sum_{i=1}^{n} \frac{H_{0}}{n} \Psi_{i}-p r^{2} \frac{\mathrm{d} z}{\mathrm{~d} S}\right) \mathrm{d} S,
\end{aligned}
$$

where $\Psi$ is the total strain energy per unit volume of the membrane, $\Omega_{0}$ is the reference region of the membrane, with 
the infinitesimal volume element $d V$ defined in that region, and $\partial \Omega_{\sigma} \subset \partial \Omega$ is the current boundary surface on which the pressure boundary condition acts. Making use of the symmetry conditions, the volume integrals can be recast into a one-dimensional form, as indicated in (9). Equation (9) is solved using the finite element method, and to find the equilibrium state, the potential energy $\Pi$ is minimised with respect to the nodal displacements using a Newton-Raphson scheme. The finite element formulation was implemented in MATLAB, and 50 quadratic line elements were used to discretise the aneurysm contour.

3.4. Physical and Numerical Data. The systolic blood pressure in a human carotid artery is about $7.0 \mathrm{kPa}$ [53], and this pressure is taken to apply for a cerebral artery in the vasculature of the Circle of Willis as well. Assuming a ratio of $120 / 80$ between the systolic and diastolic blood pressures, the pressure levels $p_{\text {sys }}=7.0 \mathrm{kPa}$ and $p_{\text {dia }}=4.7 \mathrm{kPa}$ are obtained. The radius of a middle cerebral artery is about $1.2 \mathrm{~mm}$ [25], which gives the estimation $R_{0}=1.2 \mathrm{~mm}$, and the thickness of a healthy adventitia is about $H_{0}=100 \mu \mathrm{m}$ [25]. As the model is formulated, the constants $n_{\mathrm{fb} 0}, \beta_{0}, \mu$, and $t_{\mathrm{cl}}$ appear as a single factor $n_{\mathrm{fb} 0} \beta_{0} \mu t_{\mathrm{cl}}$. This factor may be interpreted as the initial stiffness of the collagen fabric of the adventitia and may be estimated on the basis of tensile test data to $n_{\mathrm{fb} 0} \beta_{0} \mu t_{\mathrm{cl}}=3.3 \mathrm{MPa}$ [25]. The time scale is normalised by $t_{\mathrm{cl}}$, and in the numerical analyses a constant time increment of $\Delta t=0.01 t_{\mathrm{cl}}$ was used. The aneurysm profile was discretised using 30 quadratic line elements.

In general, the collagen prestretch $\lambda_{\text {pre }}$ is important for the mechanical behaviour of collagenous tissues. Fibroblasts are known to contract the existing collagen matrix when inserting new collagen. Clinical records show that some aneurysms are able to stabilise, whereas others continue to grow perpetually. Stabilisation during continued collagen turnover does indeed require prestretching of collagen fibres, and this prestretch effect could, in principle, even allow aneurysms to shrink. However, the fact that some aneurysms stabilise, whereas others continue to grow, and the fact that shrinking has not been observed clinically (at least not to the author's knowledge) indicate that this prestretching in cerebral aneurysms is close to unity. One possible reason might be that in cerebral aneurysms the collagen fabric is under such high tension that the fibroblasts are not able to contract the collagen matrix to any significant extent. In the present work we therefore assume that $\lambda_{\text {pre }}=1$.

In a healthy artery, the load is mainly carried by the media, but as the media is degraded in a developing aneurysm, the load from the blood pressure is transferred to the adventitia. The initial conditions used here correspond to an instant transfer of the load from the media to the adventitia. Thus, in the half-closed time interval $t \in(-\infty, 0]$, the membrane is taken to have existed in the reference configuration with a surface pressure $p=0$, with the collagen production rate $\dot{m}_{i}=n_{\mathrm{fb} 0} \beta_{0}$ and the deformation $C_{\mathrm{fib}}=$ $\lambda_{\text {pre }}^{2}=1$. The associated strain energy per unit reference volume for $t \leq 0$ is then $\Psi(t \leq 0)=n_{\mathrm{fb} 0} \beta_{0} \mu\left(\lambda_{\text {pre }}^{2}-1\right)^{3} t_{\mathrm{cl}}=0$.

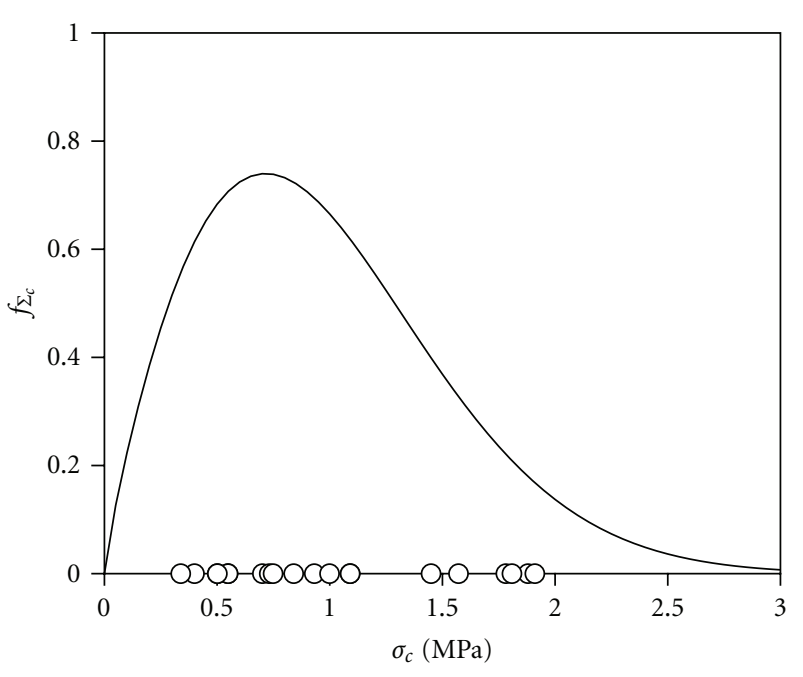

FIGURE 4: Experimental measurements $\left(\sigma_{c}^{i}\right)$ of wall strength of cerebral aneurysms (symbols) and fitted density distribution $f_{\Sigma_{c}}\left(\sigma_{c}\right)$ (solid line).

\section{Assessment of Probabilistic Framework}

Some measurements of the strength of tissue from cerebral aneurysms exist $[5,28,48,49]$. A Weibull distribution is employed for the density function $f_{\Sigma_{c}}\left(\sigma_{c}\right)$ according to

$$
f_{\Sigma_{c}}\left(\sigma_{c}\right)=\frac{k_{1}}{k_{2}}\left(\frac{\sigma_{c}}{k_{2}}\right)^{k_{1}-1} \cdot \exp \left(-\left(\frac{\sigma_{c}}{k_{2}}\right)^{k_{1}}\right),
$$

where $k_{1}$ and $k_{2}$ are constants to be fitted to the experimentally measured strength data. The two constants were determined by requiring that the mean value and variance of $f_{\Sigma_{c}}\left(\sigma_{c}\right)$ be equal to the mean and variance values of the experimental results, respectively, corresponding to the two conditions $m=\hat{m}$ and $v=\hat{v}$, where

$$
\begin{aligned}
& m=\int_{0}^{\infty} \sigma_{c} f_{\Sigma_{c}}\left(\sigma_{c}\right) d \sigma_{c}, \quad \hat{m}=\frac{1}{n_{\exp }} \sum_{i=1}^{n_{\exp }} \sigma_{c}^{i}, \\
& v=\int_{0}^{\infty}\left(\sigma_{c}-m\right)^{2} f_{\Sigma_{c}}\left(\sigma_{c}\right) d \sigma_{c}, \quad \hat{v}=\frac{1}{n_{\exp }-1} \sum_{i=1}^{n_{\text {exp }}}\left(\sigma_{c}^{i}-\hat{m}\right)^{2} .
\end{aligned}
$$

In (11), $\sigma_{c}^{i}$ denote the experimental strength values and $n_{\exp }$ is the number of experimental measurements. In Figure 4, the experimental results are shown together with the estimated Weibull distribution $\left(k_{1}=1.83\right.$ and $\left.k_{2}=1.10\right)$.

The problem for the idealised aneurysm in Section 3.3 was solved using the constitutive model in Section 3.2 and for the physical and numerical data in Section 3.4. The predicted evolution of the aneurysm is illustrated in Figure 5. In this problem, the peak stress always appears at the fundus of the aneurysm. Thus, $\sigma_{\max }$ always coincides with the maximum principal stress at the fundus. (This is in agreement with the clinical observation that cerebral aneurysms tend to rupture at the fundus.) In the present problem, the size of 


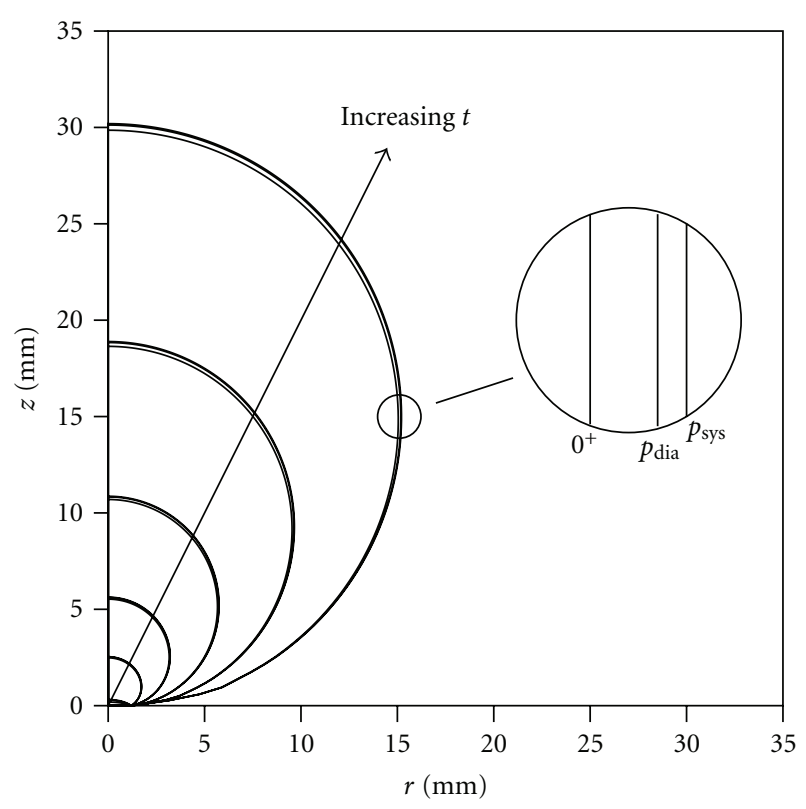

Figure 5: Predicted evolution of aneurysm geometry for $\alpha=6.3$. Aneurysm profiles are shown for the three pressure levels $p=0^{+}$, $p_{\text {dia }}, p_{\text {sys }}$ and for the times $t / t_{\mathrm{cl}}=0,23,46,69,92$, and 115 .

the aneurysm increases monotonically, and the maximum stress $\sigma_{\max }$ may be written as a function of $z_{f}$, defined as the fundus displacement in the $z$-direction. We may then express the maximum stress as $\sigma_{\max }=\sigma_{\max }\left(z_{f}\right)$. A scalar-valued function $h$ is now defined as $h\left(z_{f}\right)=\max \left(\sigma_{\max }(z), z \in\right.$ $\left.\left[0, z_{f}\right]\right)$. If the strength of the tissue is constant over time, the probability that an aneurysm of the size $z_{f}$ has experienced a maximum stress that exceeds the strength of the tissue can be expressed as

$$
P_{\text {rup }}=F_{\Sigma_{c}}\left(h\left(z_{f}\right)\right)=\int_{0}^{h\left(z_{f}\right)} f_{\Sigma_{c}}\left(\sigma_{c}\right) \mathrm{d} \sigma_{c}=F_{Z_{f}}\left(z_{f}\right),
$$

where $F_{Z_{f}}\left(z_{f}\right)$ is the distribution function for the new stochastic variable $Z_{f}$. The associated density function $f_{Z_{f}}\left(z_{f}\right)$ is obtained by differentiation as

$$
f_{Z_{f}}\left(z_{f}\right)=\frac{\mathrm{d} F_{Z_{f}}}{\mathrm{~d} z_{f}}=f_{\Sigma_{c}}\left(h\left(z_{f}\right)\right) \cdot \frac{\mathrm{d} h}{\mathrm{~d} z_{f}},
$$

where $f_{Z_{f}}\left(z_{f}\right)$ should be interpreted as the probability that an aneurysm will fail at the specific aneurysm size $z_{f}$.

The first clinical study to be used for evaluating the proposed probabilistic framework is a Japanese study by [54]. This study gives the size of 109 ruptured aneurysms in patients from Japan only. The only model parameter that has not yet been determined is the exponent $\alpha$ in the material production law in (4). In Figure 6, clinical results are compared to model predictions for some different values of $\alpha$. Comparisons are made in terms of $f_{Z_{f}}\left(z_{f}\right)$ and $F_{Z_{f}}\left(z_{f}\right)$ from clinical results and model predictions, respectively. Shaded areas indicate clinical results. The shaded bars in Figure 6(a) have been obtained by first dividing the number of ruptured aneurysms in each size range by the total number of ruptured aneurysms included in the study and then by the size of the range itself. The shaded diagram in Figure 6(b) signifies the accumulated risk of rupture in the clinical results as a function of aneurysm size and is obtained by integration of the shaded areas in (a). The value $\alpha=6.1$ yields the best agreement between clinical results and model predictions. It is evident from Figure 6(b) that the model is able to reproduce the clinical accumulated risk of rupture $P_{\text {rup }}$ well.

Studies in [1] also compile clinical results on ruptured and unruptured aneurysms, including the size of ruptured aneurysms. Their study include data for 79 ruptured aneurysms and is not limited to Japanese patients but includes patients from different parts of the world. In Figure 7, clinical results from this study are compared to model predictions for some different values of $\alpha$. Comparisons are again made in terms of $f_{Z_{f}}\left(z_{f}\right)$ and $F_{Z_{f}}\left(z_{f}\right)$ from clinical results and model predictions, respectively. In this case, the value $\alpha=6.3$ enables the best agreement between clinical results and model predictions. Shaded areas again indicate clinical results. Once again the model is able to capture the accumulated risk of aneurysm rupture in Figure 7(b) well.

\section{Discussion}

The first mechanically based probabilistic framework for assessment of the rupture risk of cerebral aneurysms is proposed. The methodology is based on the idea that the strength of cerebral aneurysmal tissue can be described by a statistical distribution and that by comparing the maximum wall stress from a structural analysis of the aneurysm with this strength distribution, a rupture risk can be obtained. The evolution of the risk of rupture with aneurysm size was predicted, and the constitutive model of the growing tissue is then pivotal. Several models for the constitutive behaviour of aneurysmal tissues exist in the literature, for example, [47, 51, 55-61], but the approach proposed by [47] was adopted.

Several studies exist where clinicians try to correlate the risk of aneurysm rupture with such factors as aneurysm size and aneurysm location. Information about the patientsuch as age, gender, and ethnicity - may also be correlated with rupture risk. However, since aneurysm rupture basically is a mechanical phenomenon, the assessment should ideally be performed by use of the mechanical fields in the aneurysm wall and the mechanical strength of the wall. The scheme proposed in the present paper is a first attempt to accomplish this.

For metallic materials, the risk of brittle fracture is often predicted by use of probabilistic approaches based on the Weibull distribution and a weakest link-concept, for example, [41-46]. However, brittle failure in metals is often preceded by significant amounts of plasticity, that is, metals tend to exhibit both brittle and ductile properties. In general, biological tissues have a hierarchical microstructure, and this makes the failure mechanisms more complicated than in metals. Soft biological tissues exhibit ductile properties, but failure in soft biological tissues can be expected to be strongly affected by material defects, such as irregularities in the collagen fabric. Thus, even though soft biological tissues are 


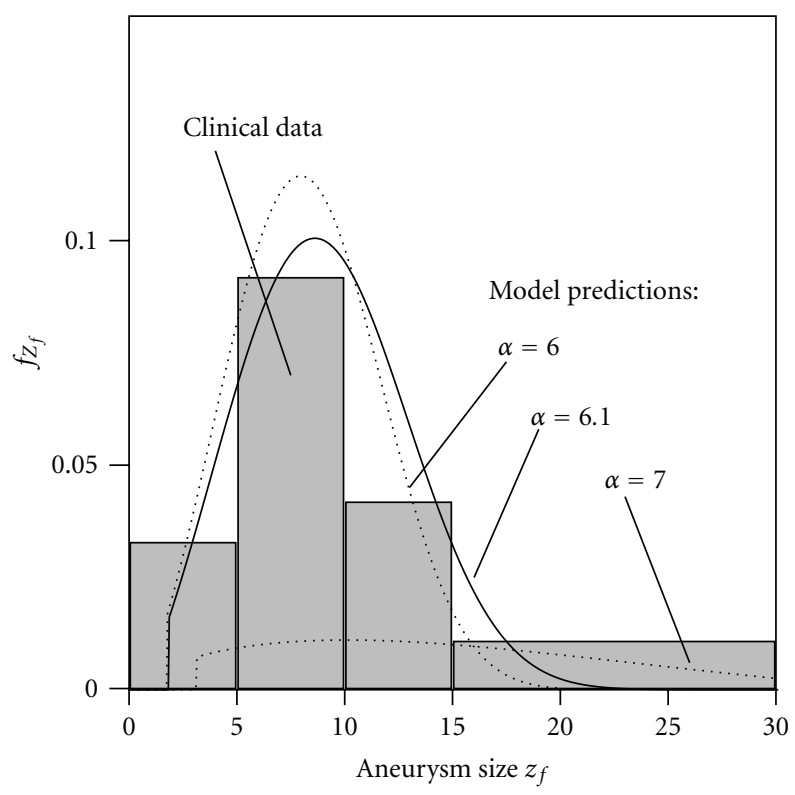

(a)

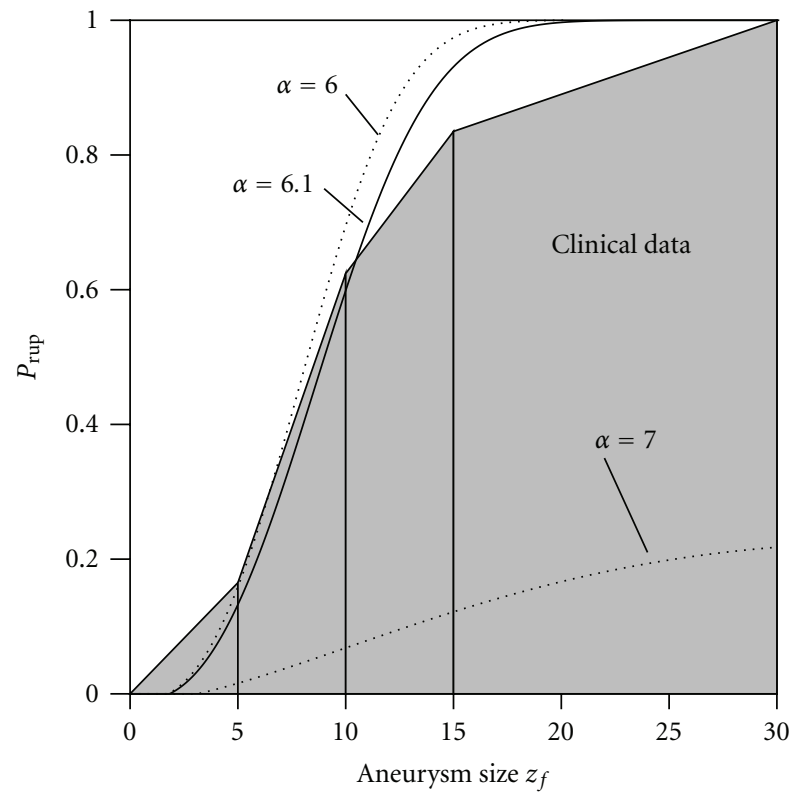

(b)

Figure 6: Comparison between clinical data from [54] and predicted risk of aneurysm rupture. Total number of ruptured aneurysms in clinical study: 109. Shaded bars and areas indicate clinical results, and lines indicate model predictions. (a) Density function for risk of rupture versus aneurysm size. (b) Rupture risk (distribution function) versus aneurysm size. The parameter $\alpha$ is the exponent in the evolution law for collagen production, see (4).

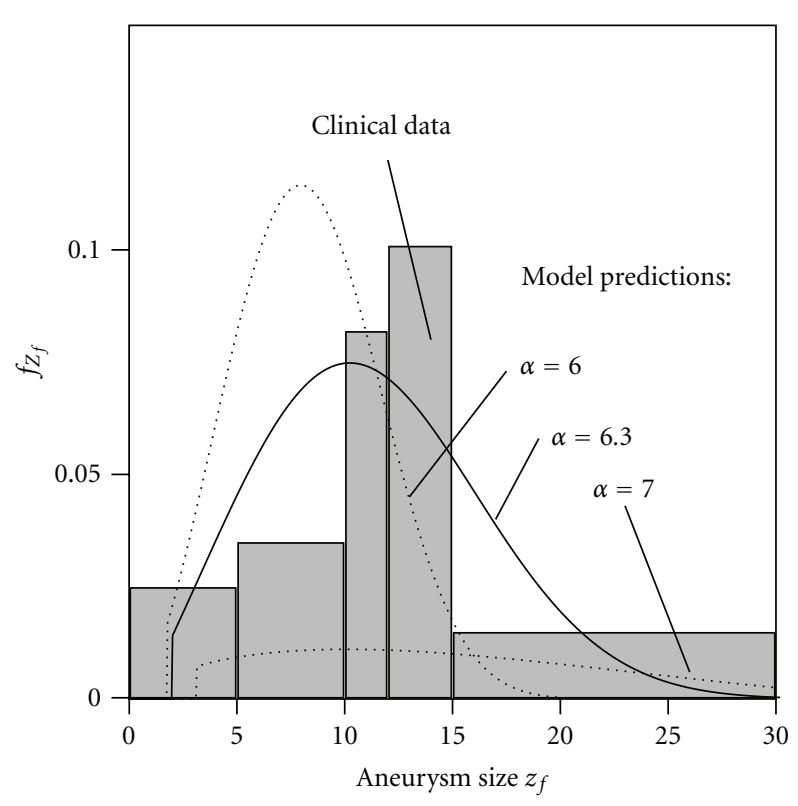

(a)

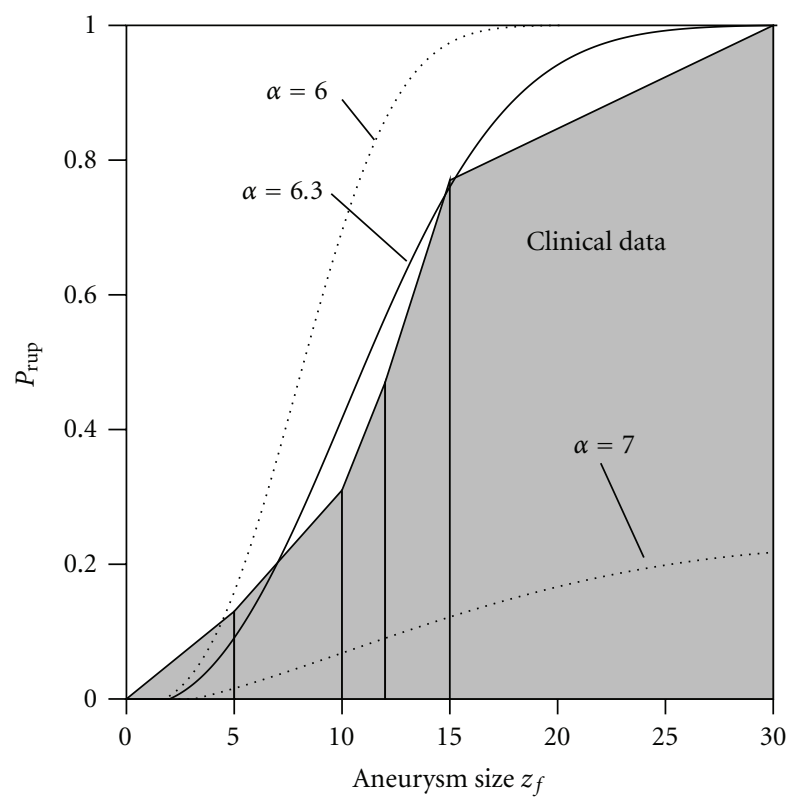

(b)

Figure 7: Comparison between clinical data from [1] and predicted risk of aneurysm rupture. Total number of ruptured aneurysms in clinical study: 79. Shaded bars and areas indicate clinical results, and lines indicate model predictions. (a) Density function for risk of rupture versus aneurysm size. (b) Rupture risk (distribution function) versus aneurysm size.

not perfectly brittle, we here make the same assumption and simplification that is generally made in fracture mechanics of metals, that a Weibull distribution may be used to represent the distribution of the strength of the material.
Besides the Weibull distribution, the Gaussian distribution is an obvious candidate for characterising the statistical distribution of material strength. For very low failure probabilities, these two distributions may give quite different 
model behaviours. However, since medical intervention by itself is a risk factor, it may be conjectured that a substantial failure risk must be present before medical intervention becomes an option.

It has been observed clinically, that cerebral aneurysms tend to rupture at the fundus $[4,8]$, and all parts of the aneurysm do not seem to be eligible for initiation of aneurysm rupture. The present framework is therefore not based on a weakest link approach, and only the point on the aneurysm surface that experiences the highest stress is considered when evaluating the risk of rupture. Failure of the aneurysm leads to a subarachnoid hemorrhage but not necessarily to a complete failure of the whole aneurysm sac.

In the constitutive model, there are a number of parameters that need to be determined. The factor $n_{\mathrm{fb} 0} \beta_{0} \mu t_{\mathrm{cl}}$ appears and is related to the stiffness of the collagen fabric in the reference state. Thus, it is not necessary to determine the parameters $n_{\mathrm{fb} 0}, \beta_{0}, \mu$, and $t_{\mathrm{cl}}$ separately. In the present computations, the original healthy adventitia was taken as the reference state, but in principle, any state of an evolving aneurysm can be chosen as reference state. In the present analysis, we applied $\lambda_{\text {pre }}=1$. This implies that the fibroblasts are unable to contract the collagen fabric when depositing new collagen into the existing network. The reason for this would be that the aneurysm wall is under such an extreme tension compared to healthy tissues. The exponent $\alpha$ governs the collagen production rate. When the theoretical model was compared with clinical results, the model was fitted by adjusting $\alpha$. The model was then able to reproduce the clinical results well, both in terms of density function and accumulated rupture risk (distribution function). Similar values of $\alpha$ were attained for the two clinical studies used (6.1 and 6.3), and when fitting the model to clinical data, $\alpha$ is expected to be in the range 5-7.

When comparing the model predictions to clinical results, the clinical results were taken from different types of arteries in the circle of Willis. In most clinical studies, results for ruptured aneurysms are not presented separately for different arteries. The physical data used in the theoretical modelling were, however, representative for the middle cerebral artery. This pertains, for example, to the radius of the parent artery, the initial thickness of the adventitia, and the mechanical stiffness of the adventitia. Some variations between different types of vessels within the circle of Willis are to be expected both in terms of geometry and in terms of mechanical properties. Studies on abdominal aortic aneurysms suggest that the strength of lesions changes during aneurysm growth and in response to physiological conditions, and the strength may also to some extent be patient specific [62]. It is therefore desirable to obtain arteryspecific values of the tissue stiffness and also of the wall strength distribution.

In the theoretical treatment, an idealised aneurysm geometry was used together with a constitutive model describing the evolution of the aneurysm. Clinical observations of the shape of real aneurysms indicate that the shape predicted by the constitutive model is reasonable; see Figure 5. It was also assumed that collagen is the only loadbearing constituent in the aneurysm wall. However, in a real aneurysm the thickness and structural composition of the wall may vary considerably. Concentration of fibroblast populations may vary, and remnants of elastin and smooth muscle may give some additional strength to the wall. When adopting the proposed probabilistic scheme in an in vivo situation, the patient-specific aneurysm geometry and the patient-specific variation of material properties should be used in the structural analysis. This may be accomplished by an inverse analysis of the type proposed by $[63,64]$ in combination with imaging techniques (e.g., MRI or CTA). In this way, patient-specific geometries and values of $n_{\mathrm{fb} 0} \beta_{0} \mu t_{\mathrm{cl}}$ and $\alpha$ could be obtained.

In summary, the first probabilistic framework for a mechanically based rupture risk assessment of cerebral aneurysms has been proposed. The method is based on the assumption that the strength of aneurysmal tissues can be described by a stochastic variable with a known distribution. A structural analysis of the aneurysm is performed, and the maximum stress is compared to the strength distribution. Based on this comparison a rupture risk is obtained. The proposed model was compared with clinical results for ruptured aneurysms in terms of rupture density and accumulated rupture risk as a function of aneurysm size. The model was able to reproduce the clinical results well. The proposed framework may potentially be used under in vivo conditions to predict the risk of rupture for diagnosed aneurysms.

\section{References}

[1] M. J. H. Wermer, I. C. van der Schaaf, A. Algra, and G. J. E. Rinkel, "Risk of rupture of unruptured intracranial aneurysms in relation to patient and aneurysm characteristics," Stroke, vol. 38, no. 4, pp. 1404-1410, 2007.

[2] P. B. Canham, R. M. Korol, H. M. Finlay et al., "Collagen organization and biomechanics of the arteries and aneurysms of the human brain," in Mechanics of Biological Tissue, G. A. Holzapfel and R. W. Ogden, Eds., pp. 307-322, Springer, Heidelberg, Germany, 2006.

[3] K. L. Mettinger, "Fibromuscular dysplasia and the brain. II. Current concept of the disease," Stroke, vol. 13, no. 1, pp. 53$58,1982$.

[4] G. Austin, S. Fisher, D. Dickson, D. Anderson, and S. Richardson, "The significance of the extracellular matrix in intracranial aneurysms," Annals of Clinical \& Laboratory Science, vol. 23, no. 2, pp. 97-105, 1993.

[5] D. J. MacDonald, H. M. Finlay, and P. B. Canham, "Directional wall strength in saccular brain aneurysms from polarized light microscopy," Annals of Biomedical Engineering, vol. 28, no. 5, pp. 533-542, 2000.

[6] A. J. Rowe, H. M. Finlay, and P. B. Canham, "Collagen biomechanics in cerebral arteries and bifurcations assessed by polarizing microscopy," Journal of Vascular Research, vol. 40, no. 4, pp. 406-415, 2003.

[7] S. Kondo, N. Hashimoto, H. Kikuchi, F. Hazama, I. Nagata, and H. Kataoka, "Apoptosis of medial smooth muscle cells in the development of saccular cerebral aneurysms in rats," Stroke, vol. 29, no. 1, pp. 181-189, 1998.

[8] W. I. Schievink, "Intracranial aneurysms," The New England Journal of Medicine, vol. 336, no. 1, pp. 28-40, 1997. 
[9] P. R. Chen, K. Frerichs, and R. Spetzler, "Natural history and general management of unruptured intracranial aneurysms," Neurosurgical Focus, vol. 17, no. 5, p. E1, 2004.

[10] V. L. Feigin, G. J. E. Rinkel, C. M. M. Lawes et al., "Risk factors for subarachnoid hemorrhage: an updated systematic review of epidemiological studies," Stroke, vol. 36, no. 12, pp. 27732780, 2005.

[11] S. Matsubara, H. Hadeishi, A. Suzuki, N. Yasui, and H. Nishimura, "Incidence and risk factors for the growth of unruptured cerebral aneurysms: observation using serial computerized tomography angiography," Journal of Neurosurgery, vol. 101, no. 6, pp. 908-914, 2004.

[12] L. Pentimalli, A. Modesti, A. Vignati et al., "Role of apoptosis in intracranial aneurysm rupture," Journal of Neurosurgery, vol. 101, no. 6, pp. 1018-1025, 2004.

[13] M. J. H. Wermer, I. C. van der Schaaf, B. K. Velthuis, A. Algra, E. Buskens, and G. J. E. Rinkel, "Follow-up screening after subarachnoid haemorrhage: frequency and determinants of new aneurysms and enlargement of existing aneurysms," Brain, vol. 128, no. 10, pp. 2421-2429, 2005.

[14] B. Weir, "Unruptured intracranial aneurysms: a review," Journal of Neurosurgery, vol. 96, no. 1, pp. 3-42, 2002.

[15] J. L. Brisman, J. K. Song, and D. W. Newell, "Cerebral aneurysms," The New England Journal of Medicine, vol. 355, no. 9, pp. 928-939, 2006.

[16] G. J. E. Rinkel, M. Djibuti, A. Algra, and J. van Gijn, "Prevalence and risk of rupture of intracranial aneurysms: a systematic review," Stroke, vol. 29, no. 1, pp. 251-256, 1998.

[17] D. O. Wiebers, J. P. Whisnant, and W. M. O'Fallon, “The natural history of unruptured intracranial aneurysms," The New England Journal of Medicine, vol. 304, no. 12, pp. 696698, 1981.

[18] Y. S. Guan and M. Q. Wang, "Endovascular embolization of intracranial aneurysms," Angiology, vol. 59, no. 3, pp. 342-351, 2008.

[19] A. I. Qureshi, V. Janardhan, R. A. Hanel, and G. Lanzino, "Comparison of endovascular and surgical treatments for intracranial aneurysms: an evidence-based review," The Lancet Neurology, vol. 6, no. 9, pp. 816-825, 2007.

[20] N. Aoki, J. R. Beck, T. Kitahara et al., "Reanalysis of unruptured intracranial aneurysm management: effect of a new international study on the threshold probabilities," Medical Decision Making, vol. 21, no. 2, pp. 87-96, 2001.

[21] H. R. Winn, J. A. Jane, J. Taylor, D. Kaiser, and A. G. W. Britz, "Prevalence of asymptomatic incidental aneurysms: review of 4568 arteriograms," Journal of Neurosurgery, vol. 96, no. 1, pp. 43-49, 2002.

[22] M. A. Jamous, S. Nagahiro, K. T. Kitazato, K. Satoh, and J. Satomi, "Vascular corrosion casts mirroring early morphological changes that lead to the formation of saccular cerebral aneurysm: an experimental study in rats," Journal of Neurosurgery, vol. 102, no. 3, pp. 532-535, 2005.

[23] R. J. Coulson, M. J. Cipolla, L. Vitullo, and N. C. Chesler, "Mechanical properties of rat middle cerebral arteries with and without myogenic tone," Journal of Biomechanical Engineering, vol. 126, no. 1, pp. 76-81, 2004.

[24] K. L. Monson, W. Goldsmith, N. M. Barbaro, and G. T. Manley, "Axial mechanical properties of fresh human cerebral blood vessels," Journal of Biomechanical Engineering, vol. 125, no. 2, pp. 288-294, 2003.

[25] K. L. Monson, W. Goldsmith, N. M. Barbaro, and G. T. Manley, "Significance of source and size in the mechanical response of human cerebral blood vessels," Journal of Biomechanics, vol. 38, no. 4, pp. 737-744, 2005.
[26] S. Scott, G. G. Ferguson, and M. R. Roach, "Comparison of the elastic properties of human intracranial arteries and aneurysms," Canadian Journal of Physiology and Pharmacology, vol. 50, no. 4, pp. 328-332, 1972.

[27] H. J. Steiger, Pathophysiology of Development and Rupture of Cerebral Aneurysms, vol. 48 of Acta Neurochirurgica Supplementum, Springer, Wien, 1990.

[28] M. Tóth, G. L. Nádasy, I. Nyáry et al., "Sterically inhomogenous viscoelastic behavior of human saccular cerebral aneurysms," Journal of Vascular Research, vol. 35, no. 5, pp. 345-355, 1998.

[29] B. K. Tóth, G. Raffai, and I. Bojtár, "Analysis of mechanical parameters of human brain aneurysm," Acta of Bioengineering and Biomechanics, vol. 7, no. 1, pp. 4-22, 2005.

[30] P. B. Canham, E. A. Talman, H. M. Finlay, and J. G. Dixon, "Medial collagen organization in human arteries of the heart and brain by polarized light microscopy," Connective Tissue Research, vol. 26, no. 1-2, pp. 121-134, 1991.

[31] P. B. Canham, H. M. Finlay, J. G. Dixon, and S. E. Ferguson, "Layered collagen fabric of cerebral aneurysms quantitatively assessed by the universal stage and polarized light microscopy," Anatomical Record, vol. 231, no. 4, pp. 579-592, 1991.

[32] P. B. Canham, P. Whittaker, S. E. Barwick, and M. E. Schwab, "Effect of pressure on circumferential order of adventitial collagen in human brain arteries," Canadian Journal of Physiology and Pharmacology, vol. 70, no. 2, pp. 296-305, 1992.

[33] P. B. Canham, H. M. Finlay, and S. Y. Tong, "Stereological analysis of the layered collagen of human intracranial aneurysms," Journal of Microscopy, vol. 183, no. 2, pp. 170-180, 1996.

[34] P. B. Canham, H. M. Finlay, J. A. Kiernan, and G. G. Ferguson, "Layered structure of saccular aneurysms assessed by collagen birefringence," Neurological Research, vol. 21, no. 7, pp. 618626, 1999.

[35] H. M. Finlay, J. G. Dixon, and P. B. Canham, "Fabric organization of the subendothelium of the human brain artery by polarized-light microscopy," Arteriosclerosis and Thrombosis, vol. 11, no. 3, pp. 681-690, 1991.

[36] H. M. Finlay, L. McCullough, and P. B. Canham, "Threedimensional collagen organization of human brain arteries at different transmural pressures," Journal of Vascular Research, vol. 32, no. 5, pp. 301-312, 1995.

[37] H. M. Finlay, P. Whittaker, and P. B. Canham, "Collagen organization in the branching region of human brain arteries," Stroke, vol. 29, no. 8, pp. 1595-1601, 1998.

[38] O. Hassler, "Scanning electron microscopy of saccular intracranial aneurysms," The American Journal of Pathology, vol. 68, no. 3, pp. 511-520, 1972.

[39] J. F. H. Smith, P. B. Canham, and J. Starkey, "Orientation of collagen in the tunica adventitia of the human cerebral artery measured with polarized light and the universal stage," Journal of Ultrastructure Research, vol. 77, no. 2, pp. 133-145, 1981.

[40] P. Whittaker, M. E. Schwab, and P. B. Canham, "The molecular organization of collagen in saccular aneurysms assessed by polarized light microscopy," Connective Tissue Research, vol. 17, no. 1, pp. 43-54, 1988.

[41] F. M. Beremin, A. Pineau, F. Mudry, J. C. Devaux, Y. D’Escatha, and P. Ledermann, "A local criterion for cleavage fracture of a nuclear pressure vessel steel," Metallurgical Transactions A, vol. 14, no. 11, pp. 2277-2287, 1983.

[42] R. H. Dodds, C. F. Shih, and T. L. Anderson, "Continuum and micromechanics treatment of constraint in fracture," International Journal of Fracture, vol. 64, no. 2, pp. 101-133, 1993. 
[43] A. Freudenthal, "Statistical approach to brittle fracture," in Fracture an Advanced Treatise, H. Liebowitz, Ed., vol. 2, pp. 592-619, Academic Press, New York, NY, USA, 1968.

[44] M. Kroon and J. Faleskog, "A probabilistic model for cleavage fracture with a length scale-influence of material parameters and constraint," International Journal of Fracture, vol. 118, no. 2, pp. 99-118, 2002.

[45] J. Faleskog, M. Kroon, and H. Öberg, "A probabilistic model for cleavage fracture with a length scale-parameter estimation and predictions of stationary crack experiments," Engineering Fracture Mechanics, vol. 71, no. 1, pp. 57-79, 2004.

[46] M. Kroon, J. Faleskog, and H. Öberg, "A probabilistic model for cleavage fracture with a length scale-parameter estimation and predictions of growing crack experiments," Engineering Fracture Mechanics, vol. 75, no. 8, pp. 2398-2417, 2008.

[47] M. Kroon and G. A. Holzapfel, "A theoretical model for fibroblast-controlled growth of saccular cerebral aneurysms," Journal of Theoretical Biology, vol. 257, no. 1, pp. 73-83, 2009.

[48] H. J. Steiger, R. Aaslid, S. Keller, and H.-J. Reulen, "Strength, elasticity and viscoelastic properties of cerebral aneurysms," Heart and Vessels, vol. 5, no. 1, pp. 41-46, 1989.

[49] B. K. Tóth, F. Nasztanovics, and I. Bojtár, "Laboratory tests for strength paramaters of brain aneurysms," Acta of Bioengineering and Biomechanics, vol. 9, no. 2, pp. 3-7, 2007.

[50] I. Fried, "Finite element computation of large rubber membrane deformations," International Journal for Numerical Methods in Engineering, vol. 18, no. 5, pp. 653-660, 1982.

[51] M. Kroon and G. A. Holzapfel, "A model for saccular cerebral aneurysm growth by collagen fibre remodelling," Journal of Theoretical Biology, vol. 247, no. 4, pp. 775-787, 2007.

[52] S. K. Kyriacou, C. Schwab, and J. D. Humphrey, "Finite element analysis of nonlinear orthotropic hyperelastic membranes," Computational Mechanics, vol. 18, no. 4, pp. 269-278, 1996.

[53] I. Chatziprodromou, A. Tricoli, D. Poulikakos, and Y. Ventikos, "Haemodynamics and wall remodelling of a growing cerebral aneurysm: a computational model," Journal of Biomechanics, vol. 40, no. 2, pp. 412-426, 2007.

[54] T. Inagawa, H. Hada, and Y. Katoh, "Unruptured intracranial aneurysms in elderly patients," Surgical Neurology, vol. 38, no. 5, pp. 364-370, 1992.

[55] S. Baek, K. R. Rajagopal, and J. D. Humphrey, "A theoretical model of enlarging intracranial fusiform aneurysms," Journal of Biomechanical Engineering, vol. 128, no. 1, pp. 142-149, 2006.

[56] J. D. Humphrey and K. R. Rajagopal, "A constrained mixture model for growth and remodeling of soft tissues," Mathematical Models and Methods in Applied Sciences, vol. 12, no. 3, pp. 407-430, 2002.

[57] M. Kroon and G. A. Holzapfel, "Modeling of saccular aneurysm growth in a human middle cerebral artery," Journal of Biomechanical Engineering, vol. 130, no. 5, pp. 1-10, 2008.

[58] A. Rachev, N. Stergiopulos, and J.-J. Meister, "Theoretical study of dynamics of arterial wall remodeling in response to changes in blood pressure," Journal of Biomechanics, vol. 29, no. 5, pp. 635-642, 1996.

[59] J. M. Ryan and J. D. Humphrey, "Finite element based predictions of preferred material symmetries in saccular aneurysms," Annals of Biomedical Engineering, vol. 27, no. 5, pp. 641-647, 1999.

[60] L. A. Taber and J. D. Humphrey, "Stress-modulated growth, residual stress, and vascular heterogeneity," Journal of Biomechanical Engineering, vol. 123, no. 6, pp. 528-535, 2001.
[61] P. N. Watton, N. A. Hill, and M. Heil, "A mathematical model for the growth of the abdominal aortic aneurysm," Biomechanics and Modeling in Mechanobiology, vol. 3, no. 2, pp. 98-113, 2004.

[62] J. P. Vande Geest, E. S. di Martino, A. Bohra, M. S. Makaroun, and D. A. Vorp, "A biomechanics-based rupture potential index for abdominal aortic aneurysm risk assessment: demonstrative application," Annals of the New York Academy of Sciences, vol. 1085, pp. 11-21, 2006.

[63] M. Kroon and G. A. Holzapfel, "Estimation of the distributions of anisotropic, elastic properties and wall stresses of saccular cerebral aneurysms by inverse analysis," Proceedings of the Royal Society A, vol. 464, no. 2092, pp. 807-825, 2008.

[64] M. Kroon and G. A. Holzapfel, "Elastic properties of anisotropic vascular membranes examined by inverse analysis," Computer Methods in Applied Mechanics and Engineering, vol. 198, no. 45-46, pp. 3622-3632, 2009. 

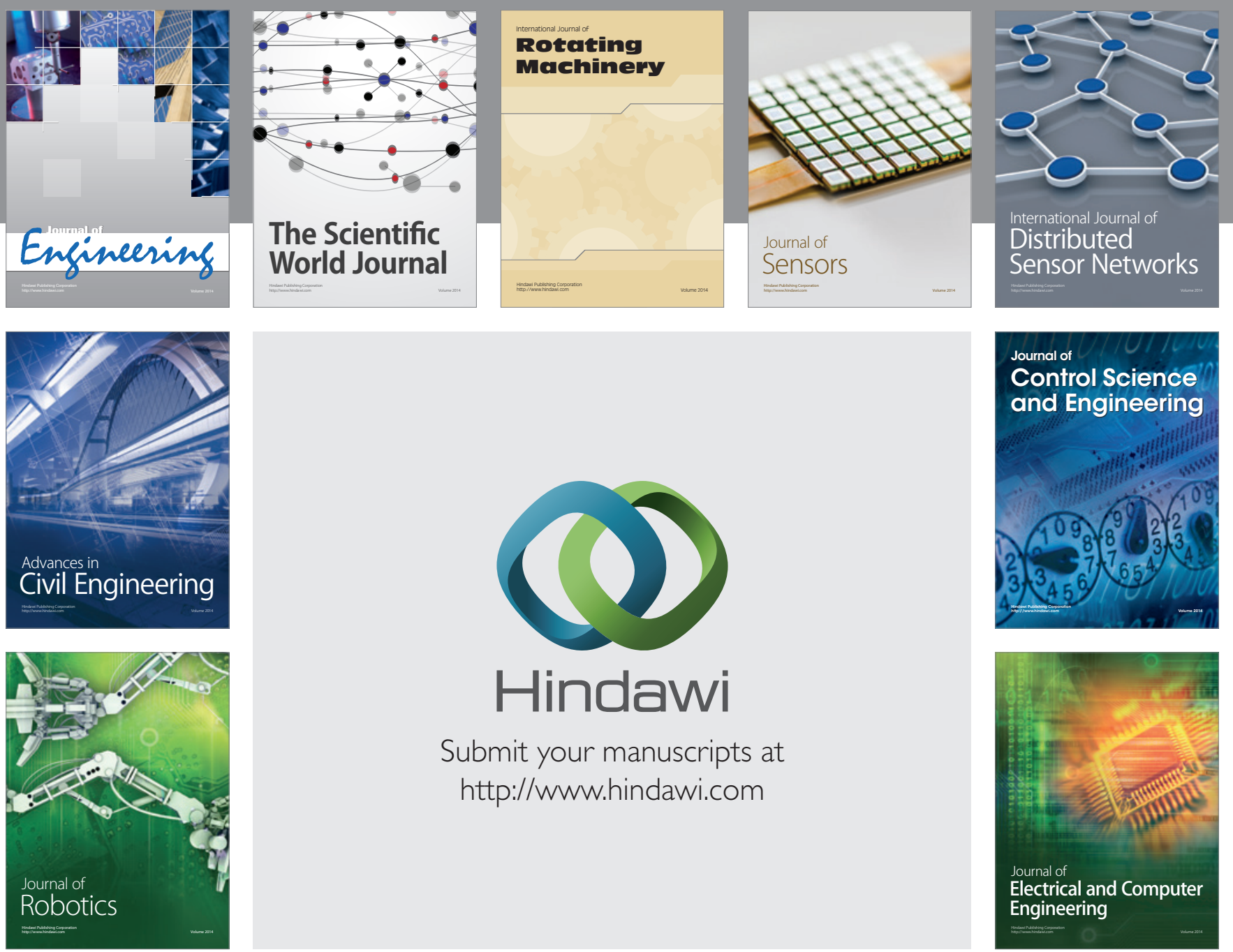

Submit your manuscripts at

http://www.hindawi.com
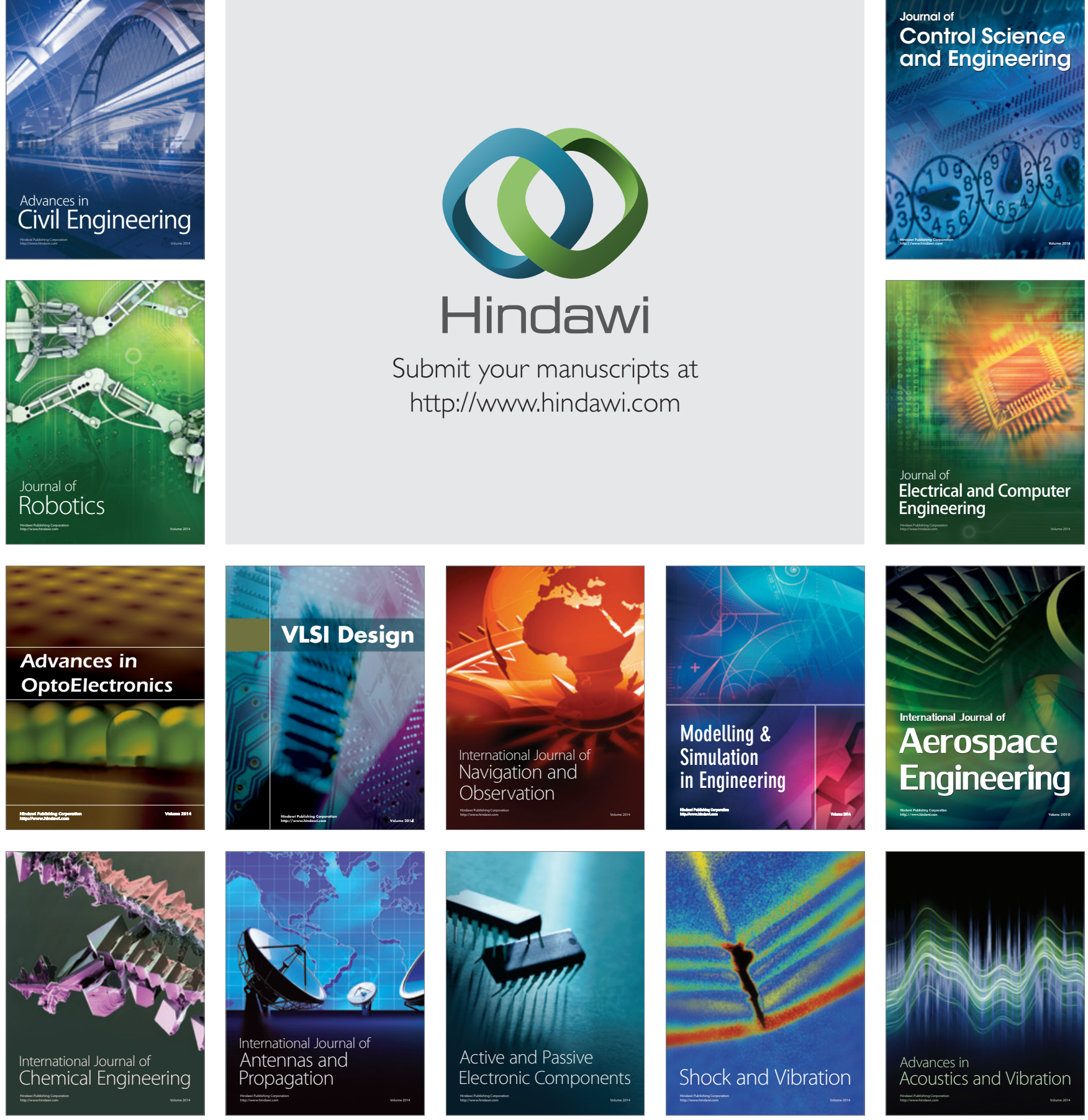